\title{
Can QT interval prolongation or dispersion detected in a positive exercise ECG test predict critical coronary artery disease?
}

\author{
Abdullah Orhan Demirtaş, Orsan Deniz Urgun
}

Cardiology Department, Adana Health Practice and Research Center, Health Science University, Adana, Turkey

Submitted: 26 October 2018

Accepted: 2 February 2019

Arch Med Sci Atheroscler Dis 2019; 4: e7-e12

DOI: https://doi.org/10.5114/amsad.2019.83299

Copyright $\odot 2019$ Termedia \& Banach

\section{Abstract}

Introduction: Exercise electrocardiography (EET) is frequently used in coronary artery disease, but the specificity of this test is very low. In the literature, parameters such as QT prolongation and QT dispersion which show coronary artery disease and arrhythmia were not sufficiently investigated using EET. The aim of this study was to investigate whether QT interval prolongation or dispersion (QT disp) in a positive EET test could predict critical coronary artery disease (CAD).

Material and methods: Patients with a positive exercise test were included in the study. Data regarding QT, QTc (corrected QT interval) and QT disp values before, during and after EET were noted. Critical coronary artery occlusions ( $\geq 70 \%$ ) was recorded from coronary angiographic images. Patients were divided into two groups (critical CAD and non-critical CAD).

Results: A total of 192 patients were found to be eligible for the study. There were 126 patients in the non-critical CAD group (group 1) and 66 patients in the critical CAD group (group 2). Recovery QTc, peak QT disp, and recovery QT disp were significantly increased in group 2 ( $p<0.001$ for each). Also, target heart rate $(p=0.012)$, basal systolic blood pressure $(p=0.005)$ and diastolic blood pressure $(p<0.001)$ were significantly higher in group 1 . Recovery QTc $(O R=1.051)$ and recovery QT disp $(O R=1.117)$ were determined as the independent predictors for critical CAD. The ROC analysis results indicated that critical CAD could be diagnosed with $90 \%$ sensitivity when the recovery QTc cut-off value was set as $404 \mathrm{~ms}$.

Conclusions: In patients with positive EET, prolonged QTC and QT disp values measured during the recovery period would predict critical CAD. Thus, the clinical accuracy of EET may be enhanced.

Key words: exercise electrocardiography, QT interval, sensitivity, coronary artery disease.

\section{Introduction}

Prevalence of coronary artery disease (CAD) is increasing in low- and moderate-risk groups where non-invasive tests are frequently used for diagnosis. Among these tests, exercise electrocardiography test (EET) is one of the most widely available, inexpensive and frequently used [1]. The EET is considered positive when ST-segment depression and/or ST-segment elevation are observed in ECG traces that result in a subsequent recommendation of an invasive test for the patient. Even though

\author{
Corresponding author: \\ Abdullah Orhan Demirtaş \\ Cardiology Department \\ Adana Health Practice \\ and Research Center \\ Health Science \\ University \\ Adana, Turkey \\ Phone: +90 5333109448 \\ E-mail: aorhandemirtas@ \\ gmail.com
}


EET is used very often, it has low sensitivity and specificity compared to other diagnostic tests [2-4]. Therefore, there is a search for additional parameters that could increase the accuracy of this test.

QT and corrected QT (QTc) interval in surface ECG is a precise parameter which gives information about the diagnosis and mortality of cardiovascular diseases [5]. QT interval can be influenced by age, cardiac ischemia, exercise, gender, smoking, diabetes mellitus, and genetic factors $[6,7]$. Heart rate increases during exercise in healthy individuals due to catecholamine discharge and thus QT interval is expected to decrease $[8,9]$. Some researchers have reported that QT dispersion would be a predictor for CAD. On the other hand, a recent study indicated that prolongation of $\mathrm{QT}$ interval is not related to $\mathrm{CAD}$ [10-12].

The aim of this study was to investigate whether QT interval prolongation or dispersion in patients with a positive exercise electrocardiography test (EET) could predict critical coronary artery disease (CAD).

\section{Material and methods}

\section{Patient selection}

Patients who were admitted to our clinic with angina pectoris and exertional dyspnea were retrospectively reviewed, and those with positive exercise test results were included. Patients with a negative exercise test were excluded from the study because coronary angiography was not performed. Patients with previous coronary artery disease, left bundle branch block, cardiac pacemaker, symptoms of pre-excitation, acute coronary syndrome, and active cardiac infection were excluded. Age, gender, systolic and diastolic blood pressures, heart rate, body mass index, the presence of any co-morbidities (hypertension, hyperlipidemia, diabetes mellitus), family history and smoking habits were noted. Gensini score of patients was also calculated.

\section{Assessment of laboratory findings}

Levels of serum glucose, urea, creatinine, sodium, potassium, uric acid, lipid panel including
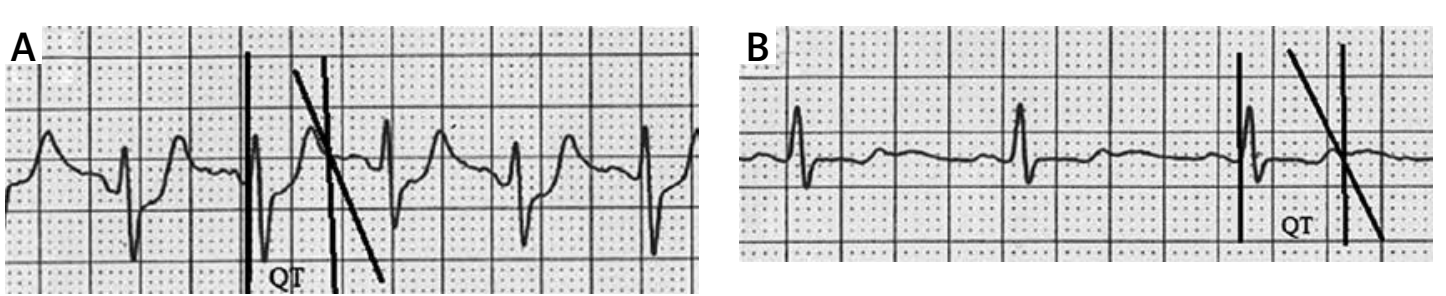

total cholesterol, high-density lipoprotein (HDL), low-density lipoprotein (LDL), very-low-density lipoprotein (VLDL) levels as well as hemoglobin, leucocyte, and neutrophil-to-lymphocyte ratio were recorded.

\section{Assessment of exercise ECG test findings}

Systolic and diastolic blood pressures, heart rate, and corrected QT (QTc) were noted. Two cardiologists assessed dispersion of QT (difference in ms between the longest and shortest QT interval) at the beginning, during the exercise (peak exercise) and at the second minute of recovery (resting period after the exercise). QTc was calculated according to the Bazett formula (Figures 1 A, B) [13]. Target heart rate was calculated by $0.8 \times(220-$ age $)$.

\section{Assessment of coronary angiographic findings}

Two independent cardiologists reviewed the coronary angiography results. Occlusion rate $\geq 70 \%$ in the left anterior descending artery, circumflex or right coronary artery or $\geq 50 \%$ in the left main coronary artery was considered critical CAD. Patients without critical CAD were labeled as group 1, and those with critical CAD were labeled as group 2.

\section{Statistical analysis}

Categorical variables were shown as numbers or percentages, and they were compared with the $\chi^{2}$ test. Continuous variables were evaluated using mean and standard deviation. The Kolmogorov-Smirnov test was used to determine whether the data were normally distributed. Independent simple $t$-test was used for analysis when the data were normally distributed and the Mann-Whitney $U$ test was used when continuous variables did not distribute normally. Binomial logistics regres$p<0.05$ to determine any independent predictors of a critical CAD. ROC analysis was performed within the independent predictors to find out their sensitivity and specificity. SPSS 20.0 (SPSS Inc., Chicago, IL, United States) was used for statistical analysis, and $p<0.05$ was regarded as statistically significant. sion analysis was performed for variables with

Figure 1. QT interval change in patients with critical CAD during EET 


\section{Results}

A total of 192 patients were eligible for the study. There were 126 patients in group 1 (mean age: $53.8 \pm 9.6$ years, $65.7 \%)$ and 66 patients in group 2 (mean age: $57.5 \pm 9.1$ years, $34.3 \%$ ). When demographic findings were compared, the Gensini score was significantly higher in group 2, while other findings were similar (Table I). Also, laboratory findings of the two groups were similar to each other (Table II). Recovery QTc, peak QT disp and recovery QT disp ( $p<0.001$ for each) were significantly higher in group 2 . Target heart rate $(p=0.012)$, basal systolic blood pressure $(p=0.005)$ and diastolic blood pressure $(p<0.001)$ were significantly higher in group 1 (Table III). In the binominal logistic regression analysis of the significant data, recovery QTc $(\mathrm{OR}=1.051,95 \% \mathrm{Cl}$ : 1.031$1.071, p<0.001)$ and recovery $\mathrm{QT}$ dispersion $(\mathrm{OR}=1.117,95 \% \mathrm{Cl}: 1.066-1.170, p<0.001)$ were found as independent predictors for critical CAD (Table IV). The ROC analysis of these independent indicators showed that the sensitivity of the test was $90 \%$ in the detection of critical CAD when the recovery QTc cut-off value was taken as $404 \mathrm{~ms}$, or the QT disp cut-off value was taken as $37 \mathrm{~ms}$ (Figures 2-4).

Table I. Comparison of demographic data of patients

\begin{tabular}{|c|c|c|c|}
\hline Parameter & Group $1(n=126)$ & Group $2(n=66)$ & $P$-value \\
\hline Age [years] & $53.8 \pm 9.6$ & $57.5 \pm 9.1$ & 0.071 \\
\hline Male gender, $n(\%)$ & $74(57.8)$ & $40(60.6)$ & 0.859 \\
\hline Systolic blood pressure $[\mathrm{mm} \mathrm{Hg}]$ & $126.4 \pm 17.5$ & $124.4 \pm 16.7$ & 0.604 \\
\hline Diastolic blood pressure [mm Hg] & $82.5 \pm 8.4$ & $83.4 \pm 7.9$ & 0.607 \\
\hline BMI $\left[\mathrm{kg} / \mathrm{m}^{2}\right]$ & $26.1 \pm 2.9$ & $26.8 \pm 1.7$ & 0.157 \\
\hline $\mathrm{HT}, n(\%)$ & $76(60.3)$ & $44(66.7)$ & 0.542 \\
\hline HPL, $n(\%)$ & $8(6.3)$ & $8(12.1)$ & 0.331 \\
\hline $\mathrm{DM}, n(\%)$ & $54(42.9)$ & $34(51.5)$ & 0.419 \\
\hline Family history, $n(\%)$ & $40(31.7)$ & $20(30.3)$ & 0.885 \\
\hline Smoking, $n(\%)$ & $74(58.7)$ & $42(63.6)$ & 0.641 \\
\hline Gensini score, $n$ & $2.6 \pm 2.8$ & $19.3 \pm 16.8$ & $<0.001$ \\
\hline
\end{tabular}

$B M I$ - body mass index, HT - hypertension, HPL - hyperlipidemia, DM - diabetes mellitus.

Table II. Comparison of laboratory findings between groups

\begin{tabular}{|lccc|}
\hline Parameter & Group $1(n=126)$ & Group 2 $(n=66)$ & $P$-value \\
\hline Glucose $[\mathrm{mg} / \mathrm{dl}]$ & $142.8 \pm 72.5$ & $166.3 \pm 94.9$ & 0.081 \\
\hline Urea $[\mathrm{mg} / \mathrm{dl}]$ & $30.1 \pm 10.4$ & $31.1 \pm 8.5$ & 0.564 \\
\hline Creatinine $[\mathrm{mg} / \mathrm{dl}]$ & $0.73 \pm 0.14$ & $0.74 \pm 0.13$ & 0.424 \\
\hline Sodium $[\mathrm{mEq} / \mathrm{l}]$ & $140.0 \pm 2.5$ & $139.4 \pm 2.5$ & 0.16 \\
\hline Potassium $[\mathrm{mEq} / \mathrm{ll}]$ & $4.3 \pm 0.3$ & $4.3 \pm 0.5$ & 0.242 \\
\hline Uric acid $[\mathrm{mg} / \mathrm{dl}]$ & $5.4 \pm 1.0$ & $5.5 \pm 0.9$ & 0.418 \\
\hline Total cholesterol [mg/dl] & $227.3 \pm 61.4$ & $232.4 \pm 57.4$ & 0.579 \\
\hline Triglyceride $[\mathrm{mg} / \mathrm{dl}]$ & $205.8 \pm 112.3$ & $224.0 \pm 145.5$ & 0.339 \\
\hline LDL cholesterol $[\mathrm{mg} / \mathrm{dl}]$ & $154.5 \pm 46.1$ & $151.3 \pm 41.3$ & 0.63 \\
\hline HDL cholesterol $[\mathrm{mg} / \mathrm{dl}]$ & $47.2 \pm 7.5$ & $46.8 \pm 8.2$ & 0.787 \\
\hline WBC $\left[10^{3} / \mu l\right]$ & $7.6 \pm 2.0$ & $8.1 \pm 2.2$ & 0.108 \\
\hline Hb $[\mathrm{g} / \mathrm{dl}]$ & $13.7 \pm 1.6$ & $13.5 \pm 1.2$ & 0.478 \\
\hline NLR & $2.4 \pm 1.1$ & $2.3 \pm 0.8$ & 0.484 \\
\hline
\end{tabular}

$L D L$ - low-density lipoprotein, HDL - high-density lipoprotein, WBC - white blood cells, Hb-hemoglobin, NLR - neutrophil-to-lymphocyte ratio. 
Table III. Comparison of EET findings between groups

\begin{tabular}{|lccc|}
\hline Parameter & Group 1 $(n=126)$ & Group 2 $(n=66)$ & $P$-value \\
\hline Basal QTc & $411.4 \pm 33.8$ & $413.3 \pm 31.3$ & 0.708 \\
\hline Peak QTc & $421.6 \pm 30.9$ & $429.6 \pm 37.9$ & 0.117 \\
\hline Recovery QTc & $406.5 \pm 26.6$ & $438.1 \pm 23.1$ & $<0.001$ \\
\hline Basal p disp & $23.6 \pm 14.9$ & $23.9 \pm 13.3$ & 0.866 \\
\hline Peak p disp & $14.3 \pm 12.3$ & $17.0 \pm 13.2$ & 0.164 \\
\hline Recovery p disp & $21.8 \pm 13.6$ & $25.3 \pm 13.5$ & 0.093 \\
\hline Basal QT disp & $34.4 \pm 13.6$ & $35.3 \pm 10.7$ & 0.657 \\
\hline Peak QT disp & $26.6 \pm 12.6$ & $38.0 \pm 14.4$ & $<0.001$ \\
\hline Recovery QT disp & $30.0 \pm 12.2$ & $42.6 \pm 9.3$ & $<0.001$ \\
\hline Heart rate recovery & $21.6 \pm 12.1$ & $15.6 \pm 5.4$ & 0.001 \\
\hline Duration of exercise & $5.9 \pm 2.4$ & $6.1 \pm 2.5$ & 0.725 \\
\hline Target heart rate & $142.2 \pm 9.9$ & $138.5 \pm 9.1$ & 0.012 \\
\hline Maximum heart rate & $149.7 \pm 16.9$ & $145.7 \pm 13.8$ & 0.095 \\
\hline First minimum heart rate & $128.1 \pm 18.7$ & $127.5 \pm 14.0$ & 0.799 \\
\hline Basal systolic BP & $133.8 \pm 14.4$ & $127.5 \pm 15.5$ & 0.005 \\
\hline Basal diastolic BP & $82.6 \pm 5.9$ & $78.0 \pm 9.5$ & $<0.001$ \\
\hline
\end{tabular}

QTC - corrected QT interval, Disp - dispersion, BP - blood pressure.

Table IV. Binominal logistics regression analysis shows independent predictors for critical CAD

\begin{tabular}{|lccc|}
\hline Parameter & Odds ratio & $95 \% \mathrm{Cl}$ & $P$-value \\
\hline Peak QT disp. & 1.022 & $0.985-1.059$ & 0.249 \\
\hline Heart rate recovery & 0.955 & $0.896-1.017$ & 0.15 \\
\hline Recovery QTc & 1.051 & $1.031-1.071$ & $<0.001$ \\
\hline Recovery QT disp & 1.117 & $1.066-1.170$ & $<0.001$ \\
\hline
\end{tabular}

QTC - corrected QT, Disp - dispersion.

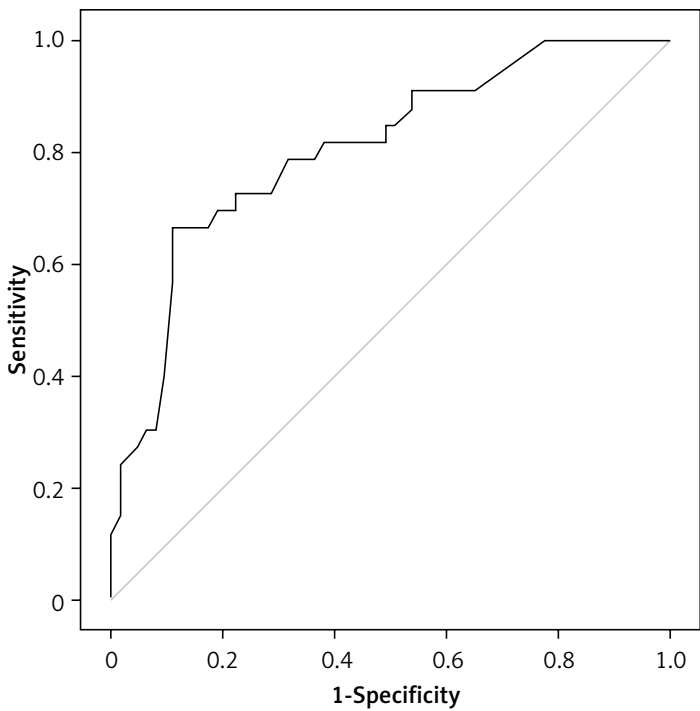

Cutt-of [ms]

404

415

425

Figure 2. ROC analysis to determine sensitivity and specificity of recovery QTC for critical coronary artery disease

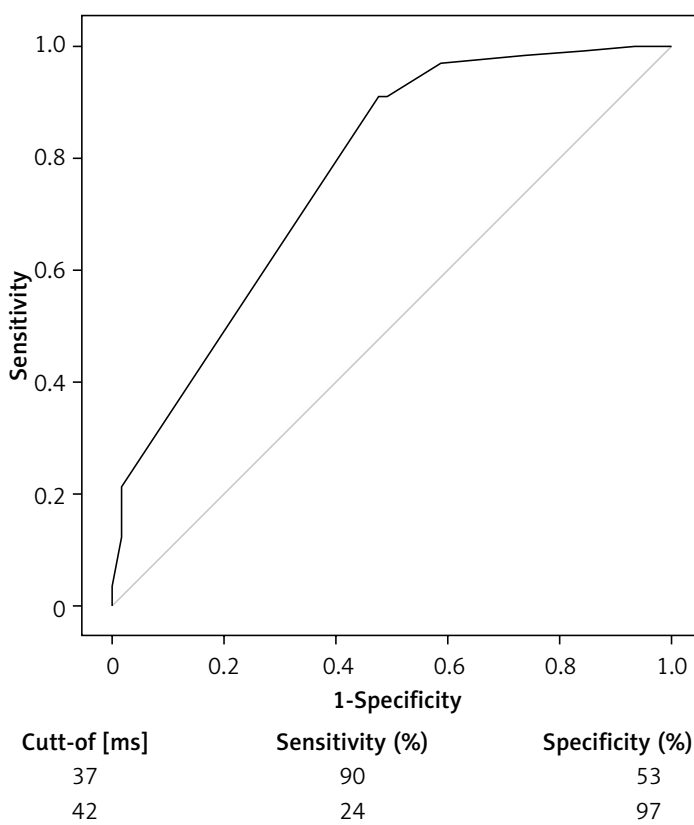

Figure 3. ROC analyses to determine sensitivity and specificity of QT dispersion for critical coronary artery disease 


\section{Discussion}

The most important finding of our study is that QT prolongation at recovery time in patients with positive EET is critical, with a very good sensitivity. QT interval is a parameter affected by physiological and/or pathological changes that occur both during cardiac depolarization and repolarization [14]. Sodium and potassium channels in cardiac muscle are responsible for cardiac depolarization and repolarization. Pathological changes of these channels might result in disruption of intracellular sodium, potassium and calcium balances [15]. Subsequently, this may be characterized by a delay in ventricular contraction and finally, expansion of the QT interval [16]. Prolongation of QT, QTc and QT disp were found to be statistically significant in the group of patients with critical CAD. Recovery QTc and recovery QT disp were also found to be predictors for critical CAD in multi-variable analysis.

As ischemia is observed in cardiac muscle cells of critical CAD patients, loss of function in intracellular ion channels occurs, leading to increase of QT interval related parameters [17]. In a previous study, CAD has been observed more frequently in patients whose QTc value was $350 \mathrm{~ms}$ at maximum heart rate during exercise ECG [12]. In contrast with this study, recovery QTc could predict $C A D$, and it was shown to be a sensitive parameter in the evaluation of EET. Therefore, we postulate that QT parameters in the recovery period may be more sensitive than QT parameters during the maximum performance.

QT hysteresis and QT hysteresis index have been reported to be significantly increased in patients who had critical CAD and underwent EET [18]. Moreover, these parameters have been shown to have high sensitivity as well as negative predictive value for EET. Although their findings are similar to ours, QT hysteresis and the QT hysteresis index are difficult to interpret. On the other hand, recovery QTC and QT disp are easier to calculate.

In a study by Barutcu et al. in which QT parameters were examined just after exercise, mortality was observed more often in the patients who had QT > 316 ms [19]. Similar to our study, it has been claimed that the early period just after exercise was more sensitive. Additionally, the same study revealed that QT interval was significantly prolonged before vs. after EET in patients with myocardial bridges (MB) compared to the normal group [19]. Despite heart muscle cells receiving blood during diastole, MB may have caused transient ischemia. We believe temporary ischemia might prolong the QT interval in these patients. Furthermore, Ozdemir et al. found that QT dispersion in female patients increased the diagnostic

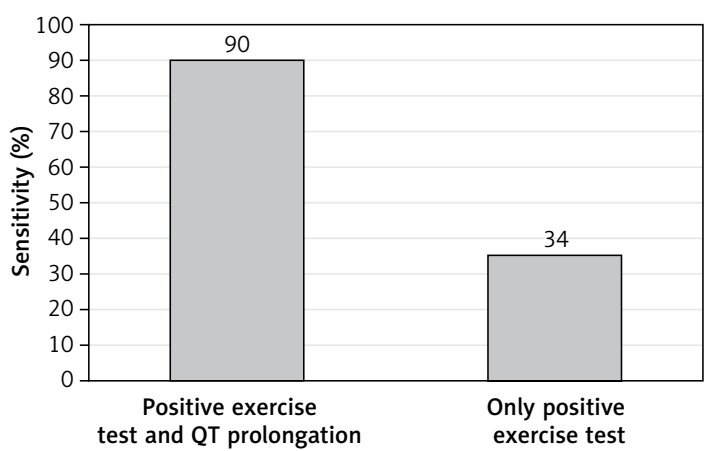

Figure 4. Comparison between sensitivity of only positive exercise test and positive exercise test-QT prolongation

value of EET [20]. As our study was not designed to address the issue and patients with negative EET were not included, information about QTc and QT disp in the patients with negative exercise test and critical CAD is lacking.

In conclusion, our results indicate that prolonged QTc and QT disp measured during the recovery period can predict critical CAD with high sensitivity. Measuring recovery QTc and QT disp is an easy and useful method to identify critical CAD in patients who have positive EET.

\section{Conflict of interest}

The authors declare no conflict of interest.

\section{References}

1. Task Force M, Montalescot G, Sechtem U, Andreotti F, et al. 2013 ESC guidelines on the management of stable coronary artery disease: the Task Force on the management of stable coronary artery disease of the European Society of Cardiology. Eur Heart J 2013; 34: 2949-3003.

2. Froelicher VF, Lehmann KG, Thomas R, et al. The electrocardiographic exercise test in a population with reduced workup bias: diagnostic performance, computerized interpretation, and multivariable prediction. Veterans Affairs Cooperative Study in Health Services \#016 (QUEXTA) Study Group. Quantitative Exercise Testing and Angiography. Ann Intern Med 1998; 128: 965-74.

3. Jeong HC, Kim I, Park KH, et al. New strategy for detection of subclinical coronary atherosclerosis in asymptomatic patients with type 2 diabetes based on cardiac multi-detector computed tomography and treadmill test. Circ J 2014; 78: 671-8.

4. Miranda CP, Liu J, Kadar A, et al. Usefulness of exercise-induced ST-segment depression in the inferior leads during exercise testing as a marker for coronary artery disease. Am J Cardiol 1992; 69: 303-7.

5. Crow RS, Hannan PJ, Folsom AR. Prognostic significance of corrected QT and corrected JT interval for incident coronary heart disease in a general population sample stratified by presence or absence of wide QRS complex: the ARIC Study with 13 years of follow-up. Circulation 2003; 108: 1985-9.

6. Schijvenaars BJ, van Herpen G, Kors JA. Intraindividual variability in electrocardiograms. J Electrocardiol 2008; 41: 190-6. 
7. Sohaib SM, Papacosta O, Morris RW, et al. Length of the QT interval: determinants and prognostic implications in a population-based prospective study of older men. J Electrocardiol 2008; 41: 704-10.

8. Sarma JS, Venkataraman SK, Samant DR, Gadgil U. Hysteresis in the human RR-QT relationship during exercise and recovery. Pacing Clin Electrophysiol 1987; 10: 485-91.

9. Davey P, Bateman J. Heart rate and catecholamine contribution to QT interval shortening on exercise. Clin Cardiol 1999; 22: 513-8.

10. Takase B, Masaki N, Hattori $\mathrm{H}$, et al. Usefulness of automatic QT dispersion measurement for detecting exercise-induced myocardial ischemia. Anatol J Cardiol 2009; 9: 189-95.

11. Ulgen MS, Karadede A, Alan S, et al. Contribution of the peak exercise QT dispersion to the accuracy of an exercise test during the evaluation of coronary artery disease. Acta Cardiol 2000; 55: 335-9.

12. Akyuz A, Alpsoy S, Akkoyun DC, et al. Maximal exercise-corrected qt as a predictor of coronary artery disease: comparison of simpler heart rate corrections. Korean Circ J 2013; 43: 655-63.

13. Ahnve S. Correction of the QT interval for heart rate: review of different formulas and the use of Bazett's formula in myocardial infarction. Am Heart J 1985; 109: 568-74.

14. Postema PG, Wilde AA. The measurement of the QT interval. Curr Cardiol Rev 2014; 10: 287-94.

15. Opthof T, Coronel R, Janse MJ. Is there a significant transmural gradient in repolarization time in the intact heart? Repolarization gradients in the intact heart. Circ Arrhythm Electrophysiol 2009; 2: 89-96.

16. Coronel R, de Bakker JM, Wilms-Schopman FJ, et al. Monophasic action potentials and activation recovery intervals as measures of ventricular action potential duration: experimental evidence to resolve some controversies. Heart Rhythm 2006; 3: 1043-50.

17. Roukema G, Singh JP, Meijs M, et al. Effect of exercise-induced ischemia on QT interval dispersion. Am Heart J 1998; 135: 88-92.

18. Zhang Y, Bao M, Dai M, et al. QT hysteresis index improves the power of treadmill exercise test in the screening of coronary artery disease. Circ J 2014; 78: 2942-9.

19. Barutcu I, Sezgin AT, Gullu H, et al. Exercise-induced changes in QT interval duration and dispersion in patients with isolated myocardial bridging. Int J Cardiol 2004; 94: 177-80.

20. Ozdemir K, Altunkeser BB, Aydin M, et al. New parameters in the interpretation of exercise testing in women: QTc dispersion and QT dispersion ratio difference. Clin Cardiol 2002; 25: 187-92. 